\title{
MULTIPLE CRITICAL POINTS OF PERTURBED SYMMETRIC FUNCTIONALS
}

BY

PAUL H. RABINOWITZ ${ }^{1}$

\begin{abstract}
Variational problems which are invariant under a group of symmetries often possess multiple solutions. This paper studies the effect of perturbations which are not small and which destroy the symmetry for two classes of such problems and shows how multiple solutions persist despite the perturbation.
\end{abstract}

During the past fifteen years there has been a considerable amount of research on the role of symmetry in obtaining multiple critical points of symmetric functionals both in an abstract setting and in applications to ordinary and partial differential equations. In particular, problems of the form,

$$
\left\{\begin{array}{l}
L u \equiv-\sum_{i, j=1}^{n} \frac{\partial}{\partial x_{i}}\left(a_{i j}(x) u_{x_{j}}\right)+c(x) u=p(x, u), \quad x \in \Omega, \\
u=0, \quad x \in \partial \Omega
\end{array}\right.
$$

have been studied where $L$ is uniformly elliptic with e.g. $C^{2}$ coefficients, $\Omega \subset \mathbf{R}^{n}$ is a bounded domain with a smooth boundary, and $p$ is odd in $u$. Under appropriate hypotheses on $p(x, \xi)$, in particular more rapid growth than linear as $|\xi| \rightarrow \infty$, it has been shown that $(0.1)$ possesses an unbounded sequence of solutions [1-6]. Similar existence statements have been obtained for periodic solutions of second order Hamiltonian systems of ordinary differential equations,

$$
\ddot{q}+V^{\prime}(q)=0,
$$

where $q=\left(q_{1}, \ldots, q_{n}\right)$ and $V \in C^{1}\left(\mathbf{R}^{n}, \mathbf{R}\right)$. Indeed, it has been shown that if $V$ grows at an appropriate superquadratic rate, then for any $T>0,(0.2)$ possesses an unbounded sequence of $T$ periodic solutions $[7,8]$.

Problems (0.1) and (0.2) each possess a natural symmetry. Namely $(0.1)$ is the Euler equation obtained from

$$
\int_{\Omega}\left[\frac{1}{2}\left(\sum_{i, j=1}^{n} a_{i j}(x) u_{x_{i}} u_{x_{j}}+c(x) u^{2}\right)-P(x, u)\right] d x
$$

Received by the editors May 22, 1981.

1980 Mathematics Subject Classification. Primary 35J20, 35J60, 34C25; Secondary 47H15.

'This research was sponsored in part by the Office of Naval Research under Contract No. N00014-76C-0300 and by the U. S. Army under Contract No. DAAG 29-75-C-0024. Reproduction in whole or in part is permitted for the purposes of the U. S. Government. 
where $P$, the primative of $p$, is even in $u$ and therefore the functional is invariant under the $\mathbf{Z}_{2}$ symmetry $u \rightarrow-u$. Likewise, taking e.g. $T=2 \pi$, we see $(0.2)$ is the Euler equation of the functional

$$
\int_{0}^{2 \pi}\left[\frac{1}{2}|\dot{q}|^{2}-V(q)\right] d t
$$

defined on the class of $2 \pi$ periodic functions. If $q(t) \rightarrow q(t+\theta)$ for any $\theta \in \mathbf{R}$, the functional is unchanged. Thus ( 0.4$)$ has a natural $\mathbf{R} \bmod [0,2 \pi]$ or $S^{1}$ symmetry.

An open question for problems like $(0.1)$ and $(0.2)$ has been the effect of destroying the above symmetries by perturbing the equation, e.g. by adding an inhomogeneous term $f(x)$ to the right-based side of $(0.1)$ or a $2 \pi$ periodic $n$-vector $\varphi(t)$ to the right-hand side of $(0.2)$. There has been some progress in this direction during the past few months due to Bahri and Berestycki [9], Struwe [10], Dong and $\mathrm{Li}$ [11], and Bahri [12]. In [9 and 10], the authors independently show that

$$
\left\{\begin{array}{l}
L u=p(x, u)+f(x), \quad x \in \Omega, \\
u=0, \quad x \in \partial \Omega,
\end{array}\right.
$$

possesses an unbounded sequence of weak solutions provided that $f \in L^{2}(\Omega)$ and $p$ satisfies more stringent conditions than are required for the existence of solutions of $(0.1)$. In this paper we shall show how some of the ideas from [5] in conjunction with those of [9 and 10] can be used to get somewhat better existence results for $(0.5)$. Moreover closely related arguments allow us to treat perturbations of $(0.2)$ of the form

$$
q+V^{\prime}(q)=\varphi(t) .
$$

Bahri and Berestycki [13] have also recently announced related results for $(0.6)$.

In $\S 1,(0.5)$ will be treated and $(0.6)$ will be handled in $\$ 2$. An appendix contains some topological results required for the study of $(0.6)$. We are indebted to Ed Fadell and Sufian Husseini for several helpful conversations concerning these topological matters.

1. The semilinear elliptic case. We begin by studying

$$
\left\{\begin{array}{l}
L u=p(x, u)+f(x), \quad x \in \Omega, \\
u=0, \quad x \in \partial \Omega,
\end{array}\right.
$$

where $L$ and $\Omega$ are in the introduction. We assume $p$ satisfies

$\left(\mathrm{p}_{1}\right) p \in C(\bar{\Omega} \times \mathbf{R}, \mathbf{R})$,

$\left(\mathrm{p}_{2}\right)$ there are constants $a_{1}, a_{2}>0$ such that $|p(x, \xi)| \leqslant a_{1}+a_{2}|\xi|^{s}$ where $1<s$ $<(n+2) /(n-2)$ if $n>2$ and $s$ is unrestricted if $n=1,2$,

$\left(\mathrm{p}_{3}\right)$ there are constants $\mu>2$ and $\bar{\xi}>0$ such that $0<\mu^{\prime} P(x, \xi) \equiv \mu \int_{0}^{\xi} p(x, t) d t$ $\leqslant \xi p(x, \xi)$ for $|\xi| \geqslant \bar{\xi}$,

$\left(\mathrm{p}_{4}\right) p(x,-\xi)=-p(x, \xi)$.

Under hypotheses $\left(\mathrm{p}_{1}\right)-\left(\mathrm{p}_{4}\right)$, if $f \equiv 0$, it is known that (1.1) possesses an unbounded sequence of weak solutions which can be obtained as critical points of a corresponding functional by means of minimax methods. We shall show that the 
same is true for (1.1) for arbitrary $f \in L^{2}$ provided that $s$ satisfies the more stringent condition

$$
\frac{(n+2)-(n-2) s}{n(s-1)}>\frac{\mu}{\mu-1} .
$$

For $\mu=s+1,(1.2)$ reduces to the assumption made in [9 and 10]. In [9 and 10] somewhat stronger versions of $\left(\mathrm{p}_{1}\right)$ and $\left(\mathrm{p}_{3}\right)$ also are needed as well as the requirement that $p$ behave like a positive function of $x$ times a pure power of $\xi$ at infinity.

For future reference we note that $\left(\mathrm{p}_{3}\right)$ implies there are constants $a_{3}, a_{4}, a_{5}$ such that

$$
\frac{1}{\mu}\left(\xi p(x, \xi)+a_{3}\right) \geqslant P(x, \xi)+a_{4} \geqslant a_{5}|\xi|^{\mu}
$$

for all $\xi \in \mathbf{R}$. Corresponding to (1.1) we have the functional

$$
I(u)=\int_{\Omega}\left[\frac{1}{2}\left(\sum_{i, j=1}^{n} a_{i j}(x) u_{x_{i}} u_{x_{j}}+c(x) u^{2}\right)-P(x, u)-f(x) u\right] d x
$$

Letting $E=W_{0}^{1,2}(\Omega)$ where the norm in $E$ is

$$
\|u\|=\left(\int_{\Omega_{i, j}} \sum_{i=1}^{n} a_{i j}(x) u_{x_{i}} u_{x_{j}} d x\right)^{1 / 2}
$$

hypotheses $\left(\mathrm{p}_{1}\right)-\left(\mathrm{p}_{2}\right)$ and standard results imply $I \in C^{1}(E, \mathbf{R})$ (provided $f \in L^{2}(\Omega)$ ).

The main result in this section is

TheOrem 1.5. Suppose p satisfies $\left(\mathrm{p}_{1}\right)-\left(\mathrm{p}_{4}\right), f \in L^{2}(\Omega)$, and s satisfies (1.2). Then $I$ has an unbounded sequence of critical values.

The corresponding critical points form an unbounded sequence of weak solutions of (1.1). Under additional regularity assumptions on $p$ and $f$ (e.g. $p$ and $f$ Hölder continuous in their arguments), standard regularity results imply these weak solutions are classical solutions of (1.1). After proving Theorem 1.5, a mild generalization of recent work of Dong and $\mathrm{Li}[\mathbf{1 1}]$ in which $f$ is allowed to depend also on $u$ will be mentioned.

In the course of the proof of Theorem 1.5, we will obtain a minimax characterization of critical values of $I$ albeit not a completely satisfactory one. In [9 and 10], the fact that any solution of (1.1) lies on the set of $w$ in $E$ such that

$$
\int_{\Omega}\left(\sum_{i, j=1}^{n} a_{i j}(x) w_{x_{i}} w_{x_{j}}+c(x) w^{2}\right) d x=\int_{\Omega} w(p(x, w)+f(x)) d x
$$

is exploited. On this set $I$ becomes

$$
I(u)=\int_{\Omega}\left(\frac{1}{2} u p(x, u)-P(x, u)-\frac{1}{2} f(x) u\right) d x
$$


which is bounded from below. (See also [1 and 2].) We will work with an indefinite functional. However for technical reasons $I$ will be replaced by a modified functional $J$. By way of motivation for the modification, the following lemma provides some a prior bounds for a critical point of $I$ in terms of the corresponding critical value. In what follows $a_{i}, \alpha_{j}$ repeatedly denote positive constants.

LEMma 1.8. Suppose $u$ is a critical point of $I$. Then there is a constant $a_{6}$ depending on $\|f\|_{L^{2}}$ such that

$$
\int_{\Omega}\left(P(x, u)+a_{4}\right) d x \leqslant \frac{1}{\mu} \int_{\Omega}\left(u p(x, u)+a_{3}\right) d x \leqslant a_{6}\left((I(u))^{2}+1\right)^{1 / 2} .
$$

Proof. Let $I^{\prime}(u)$ denote the Fréchet derivative of $u$. At a critical point of $I$ we have

$$
\begin{aligned}
I(u) & =I(u)-\frac{1}{2} I^{\prime}(u) u \\
& \geqslant\left(\frac{1}{2}-\frac{1}{\mu}\right) \int_{\Omega}\left(u p(x, u)+a_{3}\right) d x-\frac{1}{2}\|f\|_{L^{2}}\|u\|_{L^{2}}-a_{7}
\end{aligned}
$$

via (1.3). Using the fact that $\mu>2$ and the Hölder and Young inequalities we see for any $\varepsilon>0$,

$$
I(u) \geqslant a_{8} \int_{\Omega}\left(u p(x, u)+a_{3}\right) d x-a_{9}-\varepsilon\|u\|_{L^{\mu}}^{\mu}-\beta(\varepsilon)\|f\|_{L^{2}}^{\nu}
$$

where $\nu^{-1}+\mu^{-1}=1$ and $\beta(\varepsilon) \rightarrow \infty$ as $\varepsilon \rightarrow 0$. Choosing $\varepsilon$ so that $2 \varepsilon=\mu a_{5} a_{8},(1.3)$, (1.11) and the Schwarz inequality yield (1.9).

REMARK 1.12. The inequality (1.11) combined with $I^{\prime}(u) u=0$ leads to a bound for $\|u\|$ in terms of $I(u)$. However such an estimate will not be needed later.

To define the modified functional $J$, let $\chi \in C^{\infty}(\mathbf{R}, \mathbf{R})$ such that $\chi(t)=1$ for $t \leqslant 1, \chi(t) \equiv 0$ for $t>2$ and $-2<\chi^{\prime}<0$ for $t \in(1,2)$. For $u \in E$, set

$$
\varphi(u)=2 a_{6}\left(I^{2}(u)+1\right)^{1 / 2} \text { and } \psi(u)=\chi\left(\varphi(u)^{-1} \int_{\Omega}\left(P(x, u)+a_{4}\right) d x\right) .
$$

Let supp $\psi$ denote the support of $\psi$.

LEMMA 1.13. If $u \in \operatorname{supp} \psi$, then

$$
\left|\int_{\Omega} f u d x\right| \leqslant \alpha_{1}\left(|I(u)|^{1 / \mu}+1\right)
$$

where $\alpha_{1}$ depends on $\|f\|_{L^{2}}$.

Proof. By the Schwarz and Hölder inequalities and (1.3), for all $u \in E$

$$
\left|\int_{\Omega} f u d x\right| \leqslant\|f\|_{L^{2}}\|u\|_{L^{2}} \leqslant \alpha_{2}\|u\|_{L^{\mu}} \leqslant \alpha_{3}\left(\int_{\Omega}\left(P(x, u)+a_{4}\right) d x\right)^{1 / \mu} .
$$

If further $u \in \operatorname{supp} \psi$,

$$
\int_{\Omega}\left(P(x, u)+a_{4}\right) d x \leqslant 4 a_{6}\left(I^{2}(u)+1\right)^{1 / 2} \leqslant \alpha_{3}(|I(u)|+1)
$$

so (1.14) follows from (1.15)-(1.16). 
Now set

$$
J(u)=\int_{\Omega}\left[\frac{1}{2}\left(\sum_{i, j=1}^{n} a_{i j}(x) u_{x_{i}} u_{x_{j}}+c(x) u^{2}\right)-P(x, u)-\psi(u) f(x) u\right] d x .
$$

The main reason for introducing $J$ is the following estimate which holds for $J$ but not for $I$.

LEMMA 1.18. There is a constant $\beta_{1}$, depending on $\|f\|_{L^{2}}$ such that for $u \in E$ :

$$
|J(u)-J(-u)| \leqslant \beta_{1}\left(|J(u)|^{1 / \mu}+1\right) .
$$

Proof. By (1.17) and $\left(\mathrm{p}_{4}\right)$,

$$
|J(u)-J(-u)|=(\psi(u)+\psi(-u))\left|\int_{\Omega} f u d x\right| .
$$

Thus by Lemma 1.13,

$$
\psi(-u)\left|\int_{\Omega} f u d x\right| \leqslant \alpha_{1} \psi(-u)\left(|I(u)|^{1 / \mu}+1\right) .
$$

Since by (1.4) and (1.17),

$$
|I(u)| \leqslant|J(u)|+2\left|\int_{\Omega} f u d x\right|
$$

(1.21) implies that

$$
\psi(-u)\left|\int_{\Omega} f u d x\right| \leqslant \alpha_{2} \psi(-u)\left(|J(u)|^{1 / \mu}+\left|\int_{\Omega} f u d x\right|^{1 / \mu}+1\right) .
$$

Thus by Young's inequality as in (1.11), the $f u$ term on the right-hand side of (1.23) can be absorbed by the left-hand side. A corresponding estimate for the $\psi(u)$ term in (1.20) then yields (1.19).

REMARK 1.24. Although $I(u)$ does not satisfy (1.19) for all $u \in E$, it does for all solutions of (1.1). However we are unable to exploit this fact directly.

We shall show that large critical values of $J$ are critical values of $I$. First another technical lemma is needed.

LEMma 1.25. There are constants $M_{0}, \alpha_{0}>0$, and depending on $\|f\|_{L^{2}}$ such that whenever $M \geqslant M_{0}, J(u) \geqslant M$ and $u \in \operatorname{supp} \psi$, then $I(u) \geqslant \alpha_{0} M$.

Proof. Since by (1.4) and (1.17)

$$
I(u) \geqslant J(u)-\left|\int_{\Omega} f u d x\right|,
$$

if $u \in \operatorname{supp} \psi$, by (1.26) and (1.14)

$$
I(u)+\alpha_{1}|I(u)|^{1 / \mu} \geqslant J(u)-\alpha_{1} \geqslant M / 2
$$

for $M_{0}$ large enough. If $I(u) \leqslant 0$,

$$
\frac{\alpha_{1}^{\nu}}{\nu}+\frac{1}{\mu}|I(u)| \geqslant \alpha_{1}|I(u)|^{1 / \mu} \geqslant \frac{M}{2}+|I(u)|
$$


which is impossible if $M_{0}>2 \alpha_{1}^{\nu} \nu^{-1}$ which we can assume to be the case. Therefore $I(u)>0$ and

$$
I(u)>M / 4 \text { or } I(u) \geqslant\left(M\left(4 \alpha_{1}\right)^{-1}\right)^{\mu}
$$

which implies the lemma since $\mu>2$.

Now we can prove

LEMMA 1.29. There is a constant $M_{1}>0$ such that $J(u) \geqslant M_{1}$ and $J^{\prime}(u)=0$ implies that $J(u)=I(u)$ and $I^{\prime}(u)=0$.

Proof. It suffices to show that $\psi(u)=1$ and $\psi^{\prime}(u)=0$. By the definition of $\psi$, this will be the case if

$$
9(u)^{-1} \int_{\Omega}\left(P(x, u)+a_{4}\right) d x \leqslant 1
$$

which we will verify. Note that

$$
J^{\prime}(u) u=\int_{\Omega}\left[\sum_{i, j=1}^{n} a_{i j}(x) u_{x_{i}} u_{x_{j}}+c(x) u^{2}-u p(x, u)\right.
$$

$$
\left.-\left(\psi(u)+\psi^{\prime}(u) u\right) f u\right] d x
$$

where

$$
\begin{aligned}
& \psi^{\prime}(u) u=\chi^{\prime}\left(9(u)^{-1} \int_{\Omega}\left(P(x, u)+a_{4}\right) d x\right)
\end{aligned}
$$

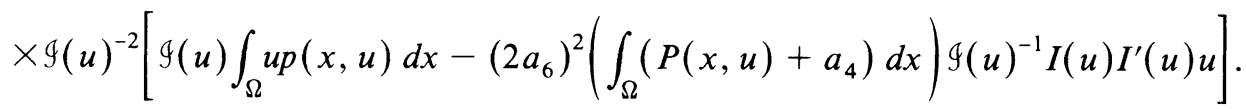

Regrouping terms shows that

$$
\begin{aligned}
J^{\prime}(u) u= & \left(1+T_{1}(u)\right) \int_{\Omega}\left(\sum_{i, j=1}^{n} a_{i j}(x) u_{x_{i}} u_{x_{j}}+c(x) u^{2}\right) d x \\
& -\left(1+T_{2}(u)\right) \int_{\Omega} u p(x, u) d x-\left(\psi(u)+T_{1}(u)\right) \int_{\Omega} f u d x
\end{aligned}
$$

where

$$
\left\{\begin{array}{l}
T_{1}(u)=\chi^{\prime}(\cdots)\left(2 a_{6}\right)^{2} 9(u)^{-3} I(u) \int_{\Omega}\left(P(x, u)+a_{4}\right) d x \int_{\Omega} f u d x, \\
T_{2}(u)=\chi^{\prime}(\cdots)\left[g(u)^{-1} \int_{\Omega} f u d x\right]+T_{1}(u) .
\end{array}\right.
$$

Form

$$
J(u)-\frac{1}{2\left(1+T_{1}(u)\right)} J^{\prime}(u) u .
$$

If $T_{1}(u)=T_{2}(u)=0$ and $\psi(u)=1$ we are precisely in the situation of (1.10) so (1.30) reduces to (1.9). If $T_{1}(u)$ and $T_{2}(u)$ are sufficiently small, the estimates of 
Lemma 1.8 carry over to this case at the expense of the factor $a_{6}$ in (1.9) being replaced by $2 a_{6}$. But that gives (1.30). Hence the lemma follows once we show $T_{1}(u), T_{2}(u) \rightarrow 0$ as $M_{1} \rightarrow \infty$.

Simple estimates show

$$
\left|T_{1}(u)\right| \leqslant\left|\chi^{\prime}(\cdots)\right| 4 a_{6} g(u)^{-1}\left|\int_{\Omega} f u d x\right| .
$$

If $u \notin \operatorname{supp} \psi, T_{1}(u)=0$. Otherwise, by Lemmas 1.13 and 1.25 ,

$$
\left|T_{1}(u)\right| \leqslant \alpha_{2} g(u)^{(1 / \mu)-1} \leqslant \alpha_{3}\left(M_{1}+1\right)^{(1 / \mu)-1},
$$

which goes to 0 as $M_{1} \rightarrow \infty$. The form of $T_{2}$ shows $T_{2}(u)$ also $\rightarrow 0$ as $M_{1} \rightarrow \infty$.

By Lemma 1.29 to prove Theorem 1.5, it suffices to show $J$ has an unbounded sequence of critical values. To begin that program, another technical result is required. Let $A_{c}=\{u \in E \mid J(u) \leqslant c\}$. We say $J$ satisfies the Palais-Smale condition (PS) if whenever a sequence $\left(u_{m}\right)$ satisfies $J\left(u_{m}\right)$ is uniformly bounded and $J^{\prime}\left(u_{m}\right) \rightarrow$ 0 , then $\left(u_{m}\right)$ is precompact.

LEMMA 1.34. $J \in C^{1}(E, \mathbf{R})$ and there is a constant $M_{2}>0$ such that $J$ satisfies (PS) on $\hat{A}_{M_{2}} \equiv\left\{u \in E \mid J(u) \geqslant M_{2}\right\}$.

Proof. Since $p$ satisfies $\left(\mathrm{p}_{1}\right),\left(\mathrm{p}_{2}\right), I \in C^{1}(E, \mathbf{R})$. (See e.g. [14].) Since $\chi \in C^{\infty}$, $\left(\mathrm{p}_{1}\right),\left(\mathrm{p}_{2}\right)$ further imply $\psi$ and therefore $J \in C^{1}(E, \mathbf{R})$. To verify (PS), we argue somewhat as in Lemma 1.29. Suppose $\left(u_{m}\right) \subset E$ with $M_{2} \leqslant J\left(u_{m}\right) \leqslant K$ and $J^{\prime}\left(u_{m}\right)$ $\rightarrow 0$. Then for all large $m$,

$$
\begin{aligned}
\rho\left\|u_{m}\right\|+K \geqslant & J\left(u_{m}\right)-\rho J^{\prime}\left(u_{m}\right) u_{m} \\
= & \left(\frac{1}{2}-\rho\left(1+T_{1}\left(u_{m}\right)\right)\right) \int_{\Omega}\left(\sum_{i, j=1}^{n} a_{i j}(x) u_{m x_{i}} u_{m x_{j}}+c u_{m}^{2}\right) d x \\
& +\rho\left(1+T_{2}\left(u_{m}\right)\right) \int_{\Omega} u_{m} p\left(x, u_{m}\right) d x-\int_{\Omega} P\left(x, u_{m}\right) d x \\
& +\left[\rho\left(\psi\left(u_{m}\right)+T_{1}\left(u_{m}\right)\right)-\psi\left(u_{m}\right)\right] \int_{\Omega} f u_{m} d x
\end{aligned}
$$

where $\rho$ is free for the moment. Thus by $\left(\mathrm{p}_{3}\right)$,

$$
\begin{aligned}
\rho\left\|u_{m}\right\|+K \geqslant & \left(\frac{1}{2}-\rho\left(1+T_{1}\left(u_{m}\right)\right)\right)\left\|u_{m}\right\|^{2} \\
& +\left(\rho\left(1+T_{2}\left(u_{m}\right)\right) \mu-1\right) \int_{\Omega}\left(P\left(x, u_{m}\right)+a_{4}\right) d x \\
& -\alpha_{2}-\left(\rho\left(1+T_{1}\left(u_{m}\right)\right)+1\right)\|f\|_{L^{2}}\left\|u_{m}\right\|_{L^{2}} \\
& -\alpha_{3}\left\|u_{m}\right\|_{L^{2}}^{2} .
\end{aligned}
$$

For $M_{2}$ sufficiently large and therefore $T_{1}, T_{2}$ sufficiently small, by $\left(\mathrm{p}_{3}\right)$ we can choose $\rho \in\left(\mu^{-1}, 2^{-1}\right)$ and $\varepsilon>0$ such that

$$
\frac{1}{2\left(1+T_{1}\left(u_{m}\right)\right)}>\rho+\varepsilon>\rho-\varepsilon>\frac{1}{\mu\left(1+T_{2}\left(u_{m}\right)\right)}
$$


uniformly in $m$. Hence (1.36) and (1.3) show

$$
\rho\left\|u_{m}\right\|+K \geqslant \varepsilon\left\|u_{m}\right\|^{2}+\frac{\varepsilon}{2} \mu a_{5}\left\|u_{m}\right\|_{L^{\mu}}^{\mu}-\alpha_{2}-\alpha_{4}\left\|u_{m}\right\|-\alpha_{3}\left\|u_{m}\right\|_{L^{2}}^{2}
$$

Using the Hölder and Young inequalities again as in (1.11) we get

$$
\rho\left\|u_{m}\right\|+K \geqslant \varepsilon\left\|u_{m}\right\|^{2}+\alpha_{5}\left\|u_{m}\right\|_{L^{\mu}}^{\mu}-\alpha_{4}\left\|u_{m}\right\|-\alpha_{6}
$$

which implies $\left\{u_{m}\right\}$ is bounded in $E$.

Since

$$
J^{\prime}\left(u_{m}\right)=\left(1+T_{1}\left(u_{m}\right)\right) u_{m}-\mathscr{P}\left(u_{m}\right)
$$

where $\mathcal{P}$ is compact-see e.g. [5] - for $M_{2}$ large enough $\left|T_{1}\left(u_{m}\right)\right| \leqslant \frac{1}{2}$ and therefore $\left(u_{m}\right)$ bounded and $J^{\prime}\left(u_{m}\right) \rightarrow 0$ implies $\left(1+T_{1}\left(u_{m}\right)\right)^{-1} \mathscr{P}\left(u_{m}\right)$ converges along a subsequence. Hence (1.40) shows $\left(u_{m}\right)$ does also and (PS) is verified.

Now we can show $J$ has an unbounded sequence of critical values. Let $0<\lambda_{1}<$ $\lambda_{2} \leqslant \cdots \leqslant \lambda_{k} \leqslant \cdots$ denote the eigenvalues of

$$
\left\{\begin{array}{l}
(L-c) v=\lambda v, \quad x \in \Omega, \\
v=0, \quad x \in \partial \Omega,
\end{array}\right.
$$

and $v_{1}, v_{2}, \cdots$ denote corresponding eigenfunctions normalized such that $\left\|v_{k}\right\|=1$. Let $E_{k} \equiv \operatorname{span}\left\{v_{1}, \ldots, v_{k}\right\}$ and $E_{k}^{\perp}$ its orthogonal complement. By (1.3) there is an $R_{k}>0$ such that $\left.J\right|_{E_{k}} \leqslant 0$ if $\|u\| \geqslant R_{k}$. Let $B_{R}$ denote the closed ball of radius $R$ in $E, D_{k} \equiv B_{R_{k}} \cap E_{k}$, and

$$
\Gamma_{k}=\left\{h \in C\left(D_{k}, E\right) \mid h \text { is odd and } h(u)=u \text { if }\|u\|=R_{k}\right\} \text {. }
$$

Define

$$
b_{k}=\inf _{h \in \Gamma_{k}} \max _{u \in D_{k}} J(h(u)), \quad k \in \mathbf{N} .
$$

If $f \equiv 0$ and $J$ is even, it was essentially shown in [5] that the numbers $b_{k}$ are critical values of $J$. If $f \neq 0$, that need not be the case. However we will use these numbers as the basis for a comparison argument to prove Theorem 1.5. First it will be shown that $b_{k} \rightarrow \infty$ as $k \rightarrow \infty$.

LEMMA 1.44. For all $k \in \mathbf{N}, \rho<R_{k}$, and $h \in \Gamma_{k}$,

$$
h\left(D_{k}\right) \cap \partial B_{\rho} \cap E_{k-1}^{\perp} \neq \varnothing .
$$

Proof. Let $h \in \Gamma_{k}$. Consider $h^{-1}\left(B_{\rho}\right)$. Since $h$ is continuous, this is a neighborhood of 0 in $E_{k}$. Let $\theta$ denote the component of $h^{-1}\left(B_{\rho}\right)$ which contains 0 . Then $\theta \subset D_{k}, \vartheta$ is symmetric with respect to the origin, i.e. $u \in \vartheta$ implies $-u \in \theta$, and $\|h(u)\|=\rho$ on $\partial \vartheta$. Let $P_{k-1}, P_{k-1}^{\perp}$ denote respectively the orthogonal projections of $E$ onto $E_{k-1}, E_{k-1}^{\perp}$ so $h(u)=P_{k-1} h(u)+P_{k-1}^{\perp} h(u)$ for $u \in E$. Since $P_{k-1} h \in$ $C\left(\partial \vartheta, E_{k-1}\right)$ and is an odd function, by one of the versions of the Borsuk-Ulam Theorem [16], $P_{k-1} h$ has a zero $\hat{u}$ on $\partial \vartheta$. Hence $h(\hat{u})=P_{k-1}^{\perp} h(\hat{u}) \in \partial B_{\rho} \cap E_{k-1}^{\perp}$ and the proof is complete.

LEMMA 1.46. There are constants $\beta_{2}>0$ and $k_{0} \in \mathbf{N}$ depending on $\|f\|_{L^{2}}$ such that for all $k \geqslant k_{0}$,

$$
b_{k} \geqslant \beta_{2} k^{((n+2)-(n-2) s) / n(s-1)} .
$$


Proof. Let $h \in \Gamma_{k}$ and $\rho<R_{k}$. By Lemma 1.44, there is a $w \in h\left(D_{k}\right) \cap \partial B_{\rho} \cap$ $E_{k-1}^{\perp}$. Therefore

$$
\max _{u \in D_{k}} J(h(u)) \geqslant J(w) \geqslant \inf _{u \in \partial B_{\rho} \cap E_{k-1}^{\perp}} J(u) .
$$

Let $u \in \partial B_{\rho} \cap E_{k-1}^{\perp}$. Then by $\left(\mathrm{p}_{2}\right)$ and some simple estimates,

$$
J(u) \geqslant \frac{1}{2} \rho^{2}-\alpha_{2}\|u\|_{L^{2}}^{2}-\alpha_{3}\|u\|_{L^{s+1}}^{s+1}-\alpha_{4}-\|f\|_{L^{2}}\|u\|_{L^{2}} .
$$

By the Gagliardo-Nirenberg inequality [17],

$$
\|u\|_{L^{s+1}} \leqslant a_{7}\|u\|^{a}\|u\|_{L^{2}}^{-a}
$$

for all $u \in E$ where $2 a=n(s-1)(s+1)^{-1}$. Moreover if $u \in E_{k-1}^{\perp}$,

$$
\|u\|_{L^{2}} \leqslant \lambda_{k-1}^{-1 / 2}\|u\|
$$

Substituting (1.50)-(1.51) into (1.49) yields

$$
J(u) \geqslant \frac{1}{2} \rho^{2}-\frac{\alpha_{2}}{\lambda_{k}} \rho^{2}-\alpha_{3} \lambda_{k}^{-(1-a)(s+1) / 2} \rho^{s+1}-\alpha_{4}-\|f\|_{L^{2} \lambda_{k}^{-1 / 2} \rho .}
$$

Choose $k_{0}$ so large that $4 \alpha_{2} \leqslant \lambda_{k}$ and $\rho=\rho_{k}$ so that

$$
\rho_{k}=\frac{1}{8} \lambda_{k}^{((1-a) / 2)((s+1) /(s-1))} \text {. }
$$

Therefore

$$
J(u) \geqslant \frac{1}{8} \rho_{k}^{2}-\|f\|_{L^{2}} \lambda_{k}^{-1 / 2} \rho_{k}-\alpha_{4} .
$$

The asymptotic distribution of the eigenvalues of (1.41) is such that for large $k$,

$$
\lambda_{k} \geqslant \alpha_{5} k^{2 / n}
$$

for $\alpha_{5}$ independent of $k$ [18]. Combining (1.54)-(1.55) then gives the lemma.

To construct a sequence of critical values of $J$, another set of comparison values first must be defined. Let

$$
U_{k}=\left\{u=t v_{k+1}+w \mid t \in\left[0, R_{k+1}\right], w \in B_{R_{k+1}} \cap E_{k},\|u\| \leqslant R_{k+1}\right\}
$$

and

$$
\begin{aligned}
& \Lambda_{k}=\left\{H \in C\left(U_{k}, E\right)|H|_{D_{k}} \in \Gamma_{k} \text { and } H(u)=u\right. \\
& \left.\qquad \text { if }\|u\|=R_{k+1} \text { or } u \in\left(B_{R_{k+1}} \backslash B_{R_{k}}\right) \cap E_{k}\right\} .
\end{aligned}
$$

Now define

$$
c_{k}=\inf _{H \in \Lambda_{k}} \max _{u \in U_{k}} J(H(u)), \quad k \in \mathbf{N} .
$$

LEMMA 1.57. Suppose $c_{k}>b_{k} \geqslant M_{2}$. Let $\delta \in\left(0, c_{k}-b_{k}\right)$ and

$$
\Lambda_{k}(\delta)=\left\{H \in \Lambda_{k} \mid J(H) \leqslant b_{k}+\delta \text { on } D_{k}\right\} .
$$

Let

$$
c_{k}(\delta)=\inf _{H \in \Lambda_{k}(\delta)} \max _{u \in U_{k}} J(H(u)), \quad k \in \mathbf{N} .
$$

Then $c_{k}(\delta)$ is a critical value of $J$.

REMARK 1.59. Since by (1.58), (1.56), and (1.43), $c_{k}(\delta) \geqslant c_{k} \geqslant b_{k}$ and $b_{k} \rightarrow \infty$ as $k \rightarrow \infty$ by Lemma 1.46, the existence of a subsequence of $c_{k}$ 's which satisfy $c_{k}>b_{k}$ 
then guarantees an unbounded sequence of critical values of $J$ and hence Theorem 1.5. As will be seen shortly, (1.2) implies that such a sequence of $c_{k}$ 's exists.

For the proof of Lemma 1.57 we require the following standard "Deformation Theorem" $[14,19]$.

LeMma 1.60. Let $J \in C^{1}(E, \mathbf{R})$ and satisfy $(P S)$ on $\hat{A}_{M}$. Then if $c>M, \bar{\varepsilon}>0$, and $c$ is not a critical value of $J$, there exists $\varepsilon \in(0, \bar{\varepsilon})$ and $\eta \in C([0,1] \times E, E)$ such that

(1) $\eta(t, u)=u$ if $u \notin J^{-1}(c-\bar{\varepsilon}, c+\bar{\varepsilon})$,

(2) $\eta\left(1, A_{c+\varepsilon}\right) \subset A_{c-\varepsilon}$.

Proof of Lemma 1.57. Note first that by the definition of $b_{k}$ and $\Lambda_{k}, \Lambda_{k}(\delta) \neq \varnothing$. Choose $\bar{\varepsilon}=\frac{1}{2}\left(c_{k}-b_{k}-\delta\right)>0$. If $c_{k}(\delta)$ is not a critical value of $J$, there exists an $\varepsilon$ and $\eta$ as in Lemma 1.60. Choose $H \in \Lambda_{k}(\delta)$ such that

$$
\max _{U_{k}} J(H(u)) \leqslant c_{k}(\delta)+\varepsilon .
$$

Consider $\eta(1, H(u)) \in C\left(U_{k}, E\right)$. Note that if $\|u\|=R_{k+1}$ or $u \in\left(B_{R_{k+1}} \backslash B_{R_{k}}\right) \cap$ $E_{k}, J(H(u))=J(u) \leqslant 0$ so $\eta(1, H(u))=u$ by (1) of Lemma 1.60 (since we can assume $\left.b_{k}>0\right)$. Therefore $\eta(1, H) \in \Lambda_{k}$. Moreover on $D_{k}, J(H(u)) \leqslant b_{k}+\delta \leqslant$ $c_{k}-\bar{\varepsilon} \leqslant c_{k}(\delta)-\bar{\varepsilon}$ via our choice of $\delta$ and $\bar{\varepsilon}$. Therefore $\eta(1, H)=H \leqslant b_{k}+\delta$ on $D_{k}$, again by (1) of Lemma 1.60. Thus $\eta(1, H) \in \Lambda_{k}(\delta)$ and by (1.61) and (2) of Lemma 1.60 ,

$$
\max _{U_{k}} J(\eta(1, H(u))) \leqslant c_{k}(\delta)-\varepsilon,
$$

contrary to the definition of $c_{k}(\delta)$. Hence $c_{k}(\delta)$ is a critical value of $J$.

REMARK 1.63. Note that $c_{k}\left(\delta_{1}\right) \geqslant c_{k}\left(\delta_{2}\right)$ if $\delta_{1} \leqslant \delta_{2}$.

Now to complete the proof of Theorem 1.5, by Remark 1.59, it suffices to show that if $s$ satisfies (1.2) and $p$ satisfies $\left(p_{1}\right)-\left(p_{4}\right), c_{k}=b_{k}$ is not possible for all large $k$. Indeed we have

LEMMA 1.64. If $c_{k}=b_{k}$ for all $k \geqslant k_{1}$, there is a constant $\gamma=\gamma\left(k_{1}\right)$ such that

$$
b_{k} \leqslant \gamma k^{\mu / \mu-1} \text {. }
$$

Thus comparing (1.65) to (1.47), we see the inequalities are incompatible if

$$
\frac{\mu}{\mu-1}<\frac{(n+2)-s(n-2)}{n(s-1)} \text {. }
$$

But this is precisely condition (1.2) on $s$. Thus Theorem 1.5 is proved.

Proof of Lemma 1.64. Let $k \geqslant k_{1}$ and $\varepsilon>0$. Choose $H \in \Lambda_{k}$ such that

$$
\max _{u \in U_{k}} J(H(u)) \leqslant b_{k}+\varepsilon .
$$

Let $\hat{H}(u)=H(u)$ if $u \in U_{k}$ and $\hat{H}(u)=-H(-u)$ if $-u \in U_{k}$. Since $\left.H\right|_{B_{R_{k+1}}} \cap E_{k}$ is odd and continuous, $\hat{H}$ is well defined and $\hat{H} \in \Gamma_{k+1}$. Therefore

$$
b_{k+1} \leqslant \max _{D_{k+1}} J(\hat{H}(u)) .
$$

But $D_{k+1}=U_{k} \cup\left(-U_{k}\right)$ and by Lemma 1.18 and (1.66),

$$
\max _{-U_{k}} J(\hat{H}(u))=b_{k}+\varepsilon+\beta_{1}\left(\left|b_{k}+\varepsilon\right|^{1 / \mu}+1\right) .
$$


Thus (1.67)-(1.68) imply

$$
b_{k+1} \leqslant b_{k}+\varepsilon+\beta_{1}\left(\left|b_{k}+\varepsilon\right|^{1 / \mu}+1\right) .
$$

Since $\varepsilon>0$ is arbitrary,

$$
b_{k+1} \leqslant b_{k}+\beta_{1}\left(\left|b_{k}\right|^{1 / \mu}+1\right)
$$

for all $k \geqslant k_{1}$. An easy induction argument-see e.g. [9-10]-then yields (1.65).

REMARK 1.71. An analysis of Theorem 1.5 shows that by slightly modifying several of the lemmas, the following result holds:

THEOREM 1.72. Suppose p satisfies $\left(\mathrm{p}_{1}\right)-\left(\mathrm{p}_{4}\right), f(x, \xi)$ satisfies $\left(\mathrm{p}_{1}\right)$,

$$
|f(x, \xi)| \leqslant \alpha_{3}+\alpha_{4}|\xi|^{\sigma}, \quad 0 \leqslant \sigma<\mu-1,
$$

and

$$
\frac{(n+2)-(n-2) s}{n(s-1)}>\frac{\mu}{\mu-\sigma-1} .
$$

Then the equation

$$
\left\{\begin{array}{l}
L u=p(x, u)+f(x, u), \quad x \in \Omega, \\
u=0, \quad x \in \partial \Omega,
\end{array}\right.
$$

possesses an unbounded sequence of weak solutions.

Theorem 1.72 generalizes a result of Dong and $\mathrm{Li}[\mathbf{1 1}]$. We will not carry out the details.

REMARK 1.75. The question of whether or not the growth restrictions on $s$ (1.2) and (1.73) are essential for these results remains open. In a very interesting recent work [12], Bahri has given a partial answer. He proved for

$$
\left\{\begin{aligned}
-\Delta u & =|u|^{s-1} u+f(x), \quad x \in \Omega, \\
u & =0, \quad x \in \partial \Omega,
\end{aligned}\right.
$$

for the full range of $s: 1<s<(n+2)(n-2)^{-1}$ that there is an open dense set of $f \in L^{2}(\Omega)$ for which (1.76) possesses an infinite number of distinct solutions. One knows from an identity of Pohozaev [20] that even if $f \equiv 0$, the result is false in general if $s \geqslant(n+2)(n-2)^{-1}$.

2. The second order Hamiltonian system case. A result analogous to Theorem 1.5 holds for second order Hamiltonian systems. Consider such a system.

$$
\ddot{q}+V^{\prime}(q)=\varphi(t)
$$

where $q \in \mathbf{R}^{n}$ and $V$ satisfies

$\left(\mathrm{V}_{1}\right) V \in C^{1}\left(\mathbf{R}^{n}, \mathbf{R}\right)$,

$\left(\mathrm{V}_{2}\right)$ There are constants $a_{1}, a_{2}>0$ and $\nu>2$ such that

$$
|V(q)| \leqslant a_{1}+a_{2}|q|^{\nu} \quad \text { for all } q \in \mathbf{R}^{n},
$$

$\left(\mathrm{V}_{3}\right)$ There are constants $\mu>2$ and $\bar{q}>0$ such that

$$
0<\mu V(q) \leqslant q \cdot V^{\prime}(q) \text { for all }|q| \geqslant \bar{q} .
$$


In $\left(V_{3}\right)$ and elsewhere $p \cdot q$ denotes the usual inner product of two elements of $\mathbf{R}^{n}$. As in $\S 1,\left(\mathrm{~V}_{3}\right)$ implies the existence of constants $a_{3}, a_{4}, a_{5}>0$ such that

$$
\frac{1}{\mu}\left(q \cdot V^{\prime}(q)+a_{3}\right) \geqslant V(q)+a_{4} \geqslant a_{5}|q|^{\mu} \text { for all } q \in \mathbf{R}^{n} .
$$

We assume $\varphi(t)$ is periodic in $t$. Without loss of generality we can take the period to be $2 \pi$. The functional corresponding to $(2.1)$ is

$$
I(q)=\int_{0}^{2 \pi}\left[\frac{1}{2}|\dot{q}|^{2}-V(q)-\varphi \cdot q\right] d t .
$$

Hypotheses $\left(\mathrm{V}_{1}\right)-\left(\mathrm{V}_{2}\right)$ imply $I \in C^{1}(E, \mathbf{R})$ where now $E=\left(W^{1,2}\left(S^{1}\right)\right)^{n}$ with the norm in $E$ given by

Our main result is

$$
\|q\|^{2}=\int_{0}^{2 \pi}\left(|\dot{q}|^{2}+|q|^{2}\right) d t
$$

THEOREM 2.4. Suppose $V$ satisfies $\left(\mathrm{V}_{1}\right)-\left(\mathrm{V}_{3}\right), \varphi \in\left(L^{2}\left(S^{1}\right)\right)^{n}$, and

$$
\nu<4 \mu-2 \text {. }
$$

Then $I(q)$ has an unbounded sequence of critical values.

As in $\$ 1$, corresponding critical points are weak solutions of (2.1) and it is easy to show they satisfy (2.1) a.e. Moreover if $\varphi \in\left(C\left(S^{1}\right)\right)^{n}$, then these weak solutions in fact belong to $\left(C^{2}\left(S^{1}\right)\right)^{n}$.

When $\varphi \equiv 0$, it is known that Theorem 2.4 is true solely under hypotheses $\left(\mathrm{V}_{1}\right)$ and $\left(\mathrm{V}_{3}\right)$ [8]. A result like Theorem 2.4 has recently been announced by Bahri and Berestycki [13] who further require $V \in C^{2}$ and in place of (2.5) have the more stringent condition $\nu<2 \mu$.

Our proof of Theorem 2.4 closely parallels that of Theorem 1.5 . Therefore we will be somewhat sketchy in our exposition here.

LEMMA 2.6. If $q$ is a critical value of $I$, there is a constant $a_{6}$ depending on $\|\varphi\|_{L^{2}}$ such that

$$
\int_{0}^{2 \pi}\left(V(q)+a_{4}\right) d t \leqslant \frac{1}{\mu} \int_{0}^{2 \pi}\left(q \cdot V^{\prime}(q)+a_{3}\right) d t \leqslant a_{6}\left(I^{2}(q)+1\right)^{1 / 2}
$$

Proof. As in Lemma 1.8.

Now we set up a modified problem for (2.1). Let $\chi, 9$ be as in $\$ 1$ (with $I(u)$ replaced by $I(q)$ given by (2.3)). Let

$$
\psi(q)=\chi\left(q(q)^{-1} \int_{0}^{2 \pi}\left(V(q)+a_{4}\right) d t\right)
$$

and set

$$
J(q)=\int_{0}^{2 \pi}\left[\frac{1}{2}|\dot{q}|^{2}-V(q)-\psi(q) \varphi \cdot q\right] d t .
$$

Lemma 2.9. If $q \in \operatorname{supp} \psi$, then

$$
\left|\int_{0}^{2 \pi} \varphi \cdot q d t\right| \leqslant \alpha_{1}\left(|I(q)|^{1 / \mu}+1\right)
$$

where $\alpha_{1}$ depends on $\|\varphi\|_{L^{2}}$. 
Proof. As in Lemma 1.13.

For $\theta \in[0,2 \pi)$, let $\left(T_{\theta} q\right)(t)=q(t+\theta)$.

LEMMA 2.11. There is a constant $\beta_{1}$ depending on $\|\varphi\|_{L^{2}}$ such that for all $q \in E$ and $\theta \in[0,2 \pi)$,

$$
\left|J(q)-J\left(T_{\theta} q\right)\right| \leqslant \beta_{1}\left(|J(q)|^{1 / \mu}+1\right) .
$$

PROof. Observing that $\|q\|_{L^{2}}=\left\|T_{\theta} q\right\|_{L^{2}}$, the proof is essentially as in Lemma 1.18 .

LEMMA 2.13. There are constants $M_{0}, \alpha_{0}>0$ and depending on $\|\varphi\|_{L^{2}}$ such that whenever $M \geqslant M_{0}, J(q) \geqslant M$, and $q \in \operatorname{supp} \psi$, then $J(q) \geqslant \alpha_{0} M$.

Proof. As in Lemma 1.25.

LEMMA 2.14. There is a constant $M_{1}>0$ such that $J(q) \geqslant M_{1}$ and $J^{\prime}(q)=0$ implies $J(q)=I(q)$ and $I^{\prime}(q)=0$.

Proof. As in Lemma 1.29.

Let $\hat{A}_{c}=\{q \in E \mid J(q) \geqslant c\}$ and $A_{c}=\{q \in E \mid J(q) \leqslant c\}$.

LEMMA 2.15. $J \in C^{1}(E, \mathbf{R})$ and there exists a constant $M_{2}>0$ such that $J$ satisfies (PS) on $\hat{A}_{M_{2}}$.

Proof. $J \in C^{1}(E, \mathbf{R})$ follows from $\left(\mathrm{V}_{1}\right),\left(\mathrm{V}_{2}\right)$ and the smoothness and form of $\psi$. To verify (PS), we argue in a similar fashion to Lemma 1.34. As in (1.36) with $\rho$ chosen to satisfy (1.37), we get

$$
\begin{gathered}
\rho\left\|q_{m}\right\|+K \geqslant \varepsilon \int_{0}^{2 \pi}\left|\dot{q}_{m}\right|^{2} d t+\frac{\mu \varepsilon}{2} a_{5}\left\|q_{m}\right\|_{L^{\mu}}^{\mu}-a_{7}\left\|q_{m}\right\|_{L^{2}} \\
=\varepsilon\left\|q_{m}\right\|^{2}+\frac{\mu \varepsilon a_{5}}{2}\left\|q_{m}\right\|_{L^{\mu}}^{\mu}-\varepsilon\left\|q_{m}\right\|_{L^{2}}^{2}-a_{7}\left\|q_{m}\right\|_{L^{2}}-a_{8} .
\end{gathered}
$$

Hence using the Hölder and Young inequalities as in (1.38), we conclude $\left\{q_{m}\right\}$ is uniformly bounded in $E$. Writing

$$
\begin{aligned}
J^{\prime}\left(q_{m}\right) Q= & \left(1+T_{1}\left(q_{m}\right)\right) \int_{0}^{2 \pi}\left(\dot{q}_{m} \cdot \dot{Q}+q_{m} \cdot Q\right) d t \\
& -\left(1+T_{1}\left(q_{m}\right)\right) \int_{0}^{2 \pi} q_{m} \cdot Q d t+\text { lower order terms }
\end{aligned}
$$

we see

$$
J^{\prime}\left(q_{m}\right)=\left(1+T_{1}\left(q_{m}\right)\right) q_{m}+\mathscr{P}\left(q_{m}\right)
$$

where $\mathscr{P}$ is compact. Thus the argument of Lemma 1.34 shows $\left(q_{m}\right)$ has a convergent subsequence and (PS) is satisfied.

As a consequence of Lemma 2.14, in order to prove Theorem 2.4, it suffices to show $J$ has an unbounded sequence of critical values. This will be accomplished as in $\$ 1$ by a comparison argument. Let $e_{1}, \ldots, e_{n}$ denote the usual orthonormal basis in $\mathbf{R}^{n}$. Define $v_{j k}=(\sin j t) e_{k}$ and $w_{j k}=(\cos j t) e_{k}$ for $j \in \mathbf{N} \cup\{0\}$ and $1 \leqslant k \leqslant n$. These functions form an orthogonal basis for $E$. Let

$$
E_{m i}=\operatorname{span}\left\{v_{j k}, w_{j k} \mid 0 \leqslant j \leqslant m, 1 \leqslant k \leqslant i\right\}
$$


where $1 \leqslant i \leqslant n$. By (2.2) there exists $R_{m i}>0$ such that $\left.J\right|_{E_{m i}} \leqslant 0$ if $\|q\| \geqslant R_{m i}$. Let $D_{m i}=B_{R_{m i}} \cap E_{m i}$. We say $h \in C\left(D_{m i}, E\right)$ is equivariant if $h\left(T_{\theta} q\right)=T_{\theta} h(q)$ for all $\theta \in[0,2 \pi]$. Let

$$
\begin{aligned}
\Gamma_{k i}=\left\{h \in C\left(D_{k i}, E\right) \mid h \text { is equivariant and } h(q)\right. & =q \\
\text { whenever }\|q\| & \left.=R_{k i} \text { or } q \in E_{0 n}\right\} .
\end{aligned}
$$

Define

$$
b_{k i}=\inf _{h \in \Gamma_{k i}} \max _{q \in D_{k i}} J(h(q)) .
$$

Let $E_{k, i-1}^{\perp}$ denote the orthogonal complement of $E_{k, i-1}$ if $i \neq 1$ and $E_{k, 0}^{\perp} \equiv E_{k-1, n}^{\perp}$.

LEMMA 2.20. For all $k \in \mathbf{N}, 1 \leqslant i \leqslant n, \rho<R_{k i}$, and $h \in \Gamma_{k i}$,

$$
h\left(D_{k i}\right) \cap \partial B_{\rho} \cap E_{k, i-1}^{\perp} \neq \varnothing .
$$

Proof. The proof of this lemma will be carried out in the Appendix.

Lemma 2.22. There are constants $\beta_{2}>0$ and $k_{0} \in \mathbf{N}$ such that for $k \geqslant k_{0}$ and $1 \leqslant i \leqslant n$,

$$
b_{k i} \geqslant \beta_{2} k^{(\nu+2) /(\nu-2)} .
$$

Proof. If $k \geqslant 1$ and $q \in \partial B_{\rho} \cap E_{k, i-1}^{\perp}$,

$$
\|q\|_{L^{2}} \leqslant\|\dot{q}\|_{L^{2}}
$$

and therefore

$$
\|q\| \leqslant 2\|\dot{q}\|_{L^{2}} .
$$

Arguing as in (1.48)-(1.49) using $\left(\mathrm{V}_{2}\right)$ and (2.25) leads to

$$
J(q) \geqslant \frac{1}{4} \rho^{2}-a_{2}\|q\|_{L^{\nu}}^{\nu}-\alpha_{2}-\alpha_{3}\|q\|_{L^{2}} .
$$

The analogues here of (1.50)-(1.51) are

$$
\|q\|_{L^{\nu}} \leqslant a_{7}\|q\|^{(\nu-2) / 2 \nu}\|q\|_{L^{2}}^{(\nu+2) / 2 \nu}
$$

for all $q \in E$ and

$$
\|q\|_{L^{2}} \leqslant k^{-1}\|\dot{q}\|_{L^{2}}
$$

for $q \in E_{k, i-1}^{\perp}$ (unless $i=1$ in which case $k$ is replaced by $k-1$ ). Continuing as in Lemma 1.46 then yields (2.23).

Next to construct critical values of $J$, let

$$
U_{k i}=\left\{q=\tau v_{k, i+1}+Q \mid \tau \in\left[0, R_{k, i+1}\right], Q \in B_{R_{k, i+1}} \cap E_{k, i},\|q\| \leqslant R_{k, i+1}\right\}
$$

where if $i=n, v_{k, i+1} \equiv v_{k+1,1}$ and $R_{k, i+1} \equiv R_{k+1,1}$. Let

$$
\begin{array}{r}
\Lambda_{k i}=\left\{H \in C\left(U_{k i}, E\right)|H|_{D_{k i}} \in \Gamma_{k i} \text { and } H(q)=q \text { when }\|q\|=R_{k, i+1},\right. \\
\text { or } \left.q \in\left(B_{R_{k, i+1}} \backslash B_{R_{k, i}}\right) \cap E_{k i}\right\}
\end{array}
$$

and define

$$
c_{k i}=\inf _{H \in \Lambda_{k i}} \max _{q \in U_{k i}} J(H(q)) .
$$


Lemma 2.29. Suppose $c_{k i}>b_{k i} \geqslant M_{2}$. Let $\delta \in\left(0, c_{k i}-b_{k i}\right)$ and

$$
\Lambda_{k i}(\delta)=\left\{H \in \Lambda_{k i} \mid J(H) \leqslant b_{k i}+\delta \text { on } D_{k i}\right\}
$$

Set

$$
c_{k i}(\delta)=\inf _{H \in \Lambda_{k i}(\delta)} \max _{q \in U_{k i}} J(H(q)) .
$$

Then $c_{k i}(\delta)$ is a critical value of $J$.

PrOOF. Essentially as in Lemma 1.57.

LEMMA 2.31. If $c_{k i}=b_{k i}$ for all $k \geqslant k_{1}$ and $1 \leqslant i \leqslant n$, then there exists $\gamma=\gamma\left(k_{1}\right)$ such that

$$
b_{k i} \leqslant \gamma k^{\mu / \mu-1} \text {. }
$$

Proof. Let $k \geqslant k_{1}, 1 \leqslant i \leqslant n, \varepsilon>0$, and $H \in \Lambda_{k i}$ such that

$$
\max _{U_{k i}} J(H(q)) \leqslant b_{k i}+\varepsilon .
$$

Let $\hat{H}(q)=H(q)$ for $q \in U_{k i}$ and $\hat{H}\left(T_{\theta} q\right)=T_{\theta} H(q)$ for $q \in U_{k i}$. Note that $\left\{T_{\theta} U_{k i} \mid\right.$ $\theta \in[0,2 \pi]\}=D_{k, i+1}$ and by construction $\hat{H}$ is equivariant. Moreover since $H \in$ $C\left(D_{k i}, E\right), \hat{H} \in C\left(D_{k, i+1}, E\right)$ and $\hat{H}(q)=q$ if $\|q\|=R_{k, i+1}$. Therefore $\hat{H} \in \Gamma_{k, i+1}$. Now arguing as in Lemma 1.64 with Lemma 1.18 replaced by Lemma 2.11, we find

$$
b_{k, i+1} \leqslant b_{k i}+\beta_{1}\left(\left|b_{k i}\right|^{1 / \mu}+1\right)
$$

where $b_{k, i+1}=b_{k+1,1}$ if $i=n$. A slight extension of the argument of [9 or 10] then yields (2.32).

The proof of Theorem 2.4 is now immediate on comparing (2.32) to (2.13) and recalling (2.5).

REMARK 2.35. As in $\S 1$, a more general perturbation than $\varphi$ may be permitted using the above arguments. Indeed we have

THEOREM 2.36. If $V$ satisfies $\left(\mathrm{V}_{1}\right)-\left(\mathrm{V}_{3}\right), \varphi(t, q) \in C\left([0,2 \pi] \times \mathbf{R}^{n}, \mathbf{R}^{n}\right)$ is $2 \pi$ periodic in $t$ and

$$
|\varphi(t, q)| \leqslant \alpha_{3}+\alpha_{4}|q|^{\sigma}
$$

where $0 \leqslant \sigma<\mu-1$ and $\nu<4 \mu(\sigma+1)^{-1}-2$, then the system

$$
\ddot{q}+V^{\prime}(q)=\varphi(t, q)
$$

has an unbounded sequence of (classical) solutions.

We omit the details.

APPENDIX. Our goal here is to prove

Lemma 2.20. For all $k \in \mathbf{N}, k \geqslant 1,1 \leqslant i \leqslant n, \rho<R_{k i}$, and $h \in \Gamma_{k i}$,

$$
h\left(D_{k i}\right) \cap \partial B_{\rho} \cap E_{k, i-1}^{\perp} \neq \varnothing .
$$

The analogous result in $\$ 1$, Lemma 1.44 , was proved with the aid of the Borsuk-Ulam Theorem. The proof of Lemma 2.20 in turn depends on an $S^{1}$ version of the Borsuk-Ulam Theorem. In [21], the following situation was studied: Let $S^{1}$ act 
on $\mathbf{R}^{l} \times \mathbf{R}^{2 k}$ via a family of orthogonal transformations such that Fix $S^{1}=\mathbf{R}^{l} \times\{0\}$. For $j<k$ we consider $\mathbf{R}^{2 j}$ to be a subspace of $\mathbf{R}^{2 k}$ via $\mathbf{R}^{2 k}=\mathbf{R}^{2 j} \times \mathbf{R}^{2(k-j)} \supset \mathbf{R}^{2 j} \times$ $\{0\}$. It was shown in [21] that

LEMMA A-1. Let $\Omega$ be a bounded invariant neighborhood of 0 in $\mathbf{R}^{l} \times \mathbf{R}^{2 k}$ and let $f \in C\left(\partial \Omega, R^{l} \times \mathbf{R}^{2 j}\right)$ where $j<k$ and $f$ is equivariant. Suppose further that $\left.f\right|_{\left(S^{1-1} \times\{0\}\right) \cap \partial \Omega}$ is the identity. Then $\{x \in \partial \Omega \mid f(x)=0\}$ is nonempty.

REMARK. If $l=0$ and Fix $S^{1}=\{0\}$, it is easy to use e.g. the index theory of [15] to prove Lemma A-1.

We will show how to use Lemma A-1 to prove Lemma 2.20. First the special case.

LemmA A-2. Let $\mathfrak{K}=\left\{\Phi \in \Gamma_{k i} \mid \Phi\left(D_{k i}\right) \subset E_{m j}\right.$ for some $m$ and $\left.j\right\}$. Then (2.21) holds for all $\Phi \in \mathfrak{T}$.

Proof. Let $h \in \mathfrak{N}$. Then $h^{-1}\left(B_{\rho}\right)$ is a neighborhood of 0 in $E_{k i}$. Let $\Omega$ be the component of $h^{-1}\left(\partial B_{\rho}\right)$ containing 0 . Then $\Omega \subset D_{k i}$ is an invariant neighborhood of 0 in $E_{k i}$ (i.e. $x \in \Omega$ implies $T_{\theta} x \in \Omega \forall \theta \in[0,2 \pi)$ ). Let $P_{k, i-1}, P_{k, i-1}^{\perp}$ denote respectively the orthogonal projection of $E$ onto $E_{k, i-1}, E_{k, i-1}^{\perp}$ respectively. Then $P_{k, i-1} h \equiv f \in C\left(\partial \Omega, E_{k, i-1}\right)$. Since $E_{k, i-1}$ is an invariant subspace of $E, f$ is an equivariant map. Note that

$$
E_{0 n}=\left\{q \in E \mid T_{\theta} q=q \text { for all } \theta \in[0,2 \pi)\right\}=\text { Fix } S^{1} .
$$

Since $h \in \mathfrak{N}, h(q)=q=f(q)$ on $E_{0 n} \cap D_{k i}$. With some obvious identifications we have satisfied the hypotheses of Lemma A-1. Hence $f$ has a zero $Q$ on $\partial \Omega$. Consequently $h(Q)=P_{k, i-1}^{\perp} h(Q) \in \partial B_{\rho} \cap E_{k, i-1}^{\perp}$. Thus (2.21) is satisfied.

Now we can give the

Proof of Lemma 2.20. Let $h \in \Gamma_{k i}$ and $m>k$. Then $P_{m i} h \in \Re$. By Lemma A-1,

$$
P_{m i} h\left(D_{k i}\right) \cap \partial B_{\rho} \cap E_{k, i-1}^{\perp} \neq \varnothing .
$$

Therefore there is a sequence of $m$ 's $\rightarrow \infty$ and corresponding to $q_{m} \in D_{k i}$ such that

$$
P_{m i} h\left(q_{m}\right) \in \partial B_{\rho} \cap E_{k, i-1}^{\perp} .
$$

Passing to a subsequence if necessary, the compactness of $D_{k i}$ implies $q_{m} \rightarrow q \in D_{k i}$. Since

$$
\left\|h(q)-P_{m i} h\left(q_{m}\right)\right\| \leqslant\left\|h(q)-P_{m i} h(q)\right\|+\left\|P_{m i}\left(h(q)-h\left(q_{m}\right)\right)\right\| \rightarrow 0
$$

as $m \rightarrow \infty$, by (A-2)

$$
h(q) \in \partial B_{\rho} \cap E_{k, i-1}^{\perp}
$$

and (2.21) is satisfied.

\section{REFERENCES}

1. C. V. Coffman, A minimum-maximum principle for a class of nonlinear integral equations, J. Analyse Math. 22 (1969), 391-419.

2. J. Hemple, Superlinear variational boundary value problems and nonuniqueness, Thesis, Univ. of New England, Australia, 1970.

3. A. Ambrosetti, On the existence of multiple solutions for a class of nonlinear boundary value problems, Rend. Sem. Mat. Univ. Padova 49 (1973), 195-204. 
4. P. H. Rabinowitz, Variational methods for nonlinear elliptic eigenvalue problems, Indiana Univ. Math. J. 23 (1974), 729-754.

5. A. Ambrosetti and P. H. Rabinowitz, Dual variational methods in critical point theory and applications, J. Funct. Anal. 14 (1973), 349-381.

6. V. Benci, Some critical point theorems and applications, Comm. Pure Appl. Math. 33 (1980), $147-172$.

7. P. H. Rabinowitz, Periodic solutions of Hamiltonian systems, Comm. Pure Appl. Math. 31 (1978), $157-184$.

8. On large norm periodic solutions of some differential equations, Ergodic Theory and Dynamical Systems, Proc. Sympos. Yi (Maryland) (A. Katok, ed.), pp. 79-80 (to appear).

9. A. Bahri and H. Berestycki, A perturbation method in critical point theory and applications, Trans. Amer. Math. Soc. 267 (1981), 1-32.

10. M. Struwe, Infinitely many critical points for functionals which are not even and applications to superlinear boundary value problems, Manuscripta Math. 32 (1980), 335-364.

11. G.-C. Dong and S. Li, On the existence of infinitely many solutions of the Dirichlet problem for some nonlinear elliptic equations, Univ. of Wisconsin Math. Research Center Tech. Rep. N02161, Dec., 1980.

12. A. Bahri, Topological results on a certain class of functionals and applications (preprint).

13. A. Bahri and H. Berestycki, Existence d'une infinité de solutions periodiques pour certains systèmes hamiltoniens en présence d' un terme de contrainte, C. R. Acad. Sci. Sér. I 292, 315-318.

14. P. H. Rabinowitz, Variational methods for nonlinear eigenvalue problems, Proc. Sympos. on Eigenvalues of Nonlinear Problems, Edizioni Cremonese, Rome, 1974, pp. 141-195.

15. E. R. Fadell and H. H. Rabinowitz, Generalized cohomological index theories for Lie group actions with an application to bifurcation questions for Hamiltonian systems, Invent. Math. 45 (1978), 139-174.

16. J. T. Schwartz, Nonlinear functional analysis, Lecture Notes, Courant Inst. of Math. Sci., New York Univ., 1965.

17. L. Nirenberg, On elliptic partial differential equations, Ann. Scuola Norm. Sup. Pisa (3) 13 (1959), $1-48$.

18. R. Courant and D. Hilbert, Methods of mathematical physics, Vol. I, Interscience, New York, 1953.

19. D. C. Clark, A variant of the Ljusternick-Schnirelman theory, Indiana Univ. Math. J. 22 (1972), $65-74$.

20. S. I. Pohozaev, Eigenfunctions of the equation $-\Delta u+\lambda f(u)=0$, Soviet Math. Dokl. 5 (1965), $1408-1411$.

21. E. R. Fadell, S. Husseini and P. H. Rabinowitz, On $S^{1}$ versions of the Borsuk-Ulam Theorem, Univ. of Wisconsin Math. Res. Center Tech. Rep., 1981.

Department of Mathematics, University of Wisconsin, Madison, Wisconsin 53706 